\title{
Statin therapy-if at first you don't succeed...
}

The majority of patients who discontinue statin therapy owing to an adverse event are able to tolerate this medication if it is represcribed. This finding from a retrospective cohort study of patients treated at medical practices in Boston, MA, USA has been published in the Annals of Internal Medicine.

In randomized, controlled trials, the reported rate of statin-related adverse events is around 5-10\%.

Such events include muscular pain or weakness, allergic reactions and, rarely, rhabdomyolysis. However, little was known about the rate and type of statin-related events and subsequent discontinuation of these agents in routine care. Stopping statin therapy potentially poses a greater threat to patient health, through increased cardiovascular risk, than the drug-related event.

The study included 107,835 patients who received a prescription for a statin (most-commonly atorvastatin) between January 2000 and December 2008. Of these individuals, $53.1 \%$ discontinued statin therapy at least once. The most-

frequently cited reason for discontinuation was 'no longer required'. Statin-related adverse events were reported in $17.4 \%$ of patients. More than half of the 11,124 patients who discontinued therapy after an adverse event were represcribed a statin within 12 months of discontinuing the first prescription. This 'rechallenge' was successful in $>90 \%$ of these individuals, who continued to take the drug for at least another 12 months.

In their paper, the investigators propose that "many statin-related events...may either be mild enough to be tolerable or not be reproducible with other statins". They suggest that "providers should consider rechallenging patients who report statin-related events to identify those who can continue taking them".

Alexandra King

Original article Zhang, $\mathrm{H}$. et al. Discontinuation of statins in outine care settings: a cohort study. Ann. Intern. Med. 158, 526-534 (2013) 